\title{
Curcumin decreases renal triglyceride accumulation through AMPK-SREBP signaling pathway in streptozotocin-induced type 1 diabetic rats ${ }^{\text {is }}$
}

\author{
Vivian Soetikno ${ }^{\mathrm{a}, \mathrm{b}}$, Flori R. Sari ${ }^{\mathrm{a}}$, Vijayakumar Sukumaran ${ }^{\mathrm{a}}$, Arun Prasath Lakshmanan ${ }^{\mathrm{a}}$, Meilei Harima ${ }^{\mathrm{a}}$, \\ Kenji Suzuki ${ }^{\mathrm{c}}$, Hiroshi Kawachi ${ }^{\mathrm{d}}$, Kenichi Watanabe ${ }^{\mathrm{a}, *}$ \\ ${ }^{a}$ Department of Clinical Pharmacology, Faculty of Pharmaceutical Sciences, Niigata University of Pharmacy and Applied Life Sciences, Niigata City 956-8603, Japan \\ ${ }^{\mathrm{b}}$ Department of Pharmacology, Faculty of Medicine, University of Indonesia, Jakarta 10430, Indonesia \\ ${ }^{\mathrm{c}}$ Department of Gastroenterology and Hepatology, Niigata University Graduate School of Medical and Dental Sciences, Niigata 951-8510, Japan \\ ${ }^{\mathrm{d}}$ Department of Cell Biology, Institute of Nephrology, Niigata University Graduate School of Medical and Dental Sciences, Niigata 951-8510, Japan
}

Received 8 August 2011; received in revised form 20 April 2012; accepted 26 April 2012

\begin{abstract}
Diabetic kidney disease has been associated with the presence of lipid deposits. We assumed that curcumin, a polyphenol, would attenuate the tissue dyslipidemic condition through activation of 5' adenosine monophosphate (AMP)-activated protein kinase (AMPK) phosphorylation and suppression of sterol regulatory element-binding protein (SREBP)-1c in the kidney and would prevent renal progression in experimental type 1 diabetic rats. Diabetes was induced with streptozotocin (STZ) $(55 \mathrm{mg} / \mathrm{kg}$ ) by intraperitoneal injection in male Sprague-Dawley rats. Three weeks after STZ injection, rats were divided into three groups, namely, control, diabetic and diabetic treated with curcumin $(100 \mathrm{mg} / \mathrm{kg} / \mathrm{day})$ by gavage for 8 weeks. We found that curcumin decreased plasma triglyceride and the amount of renal triglyceride significantly. Furthermore, treatment of diabetic rats with curcumin increased the phosphorylation of AMPK and prevented the increased renal expression of SREBP-1c and, as a result, decreased the expression of acetyl CoA carboxylase and fatty acid synthase as well as adipose differentiation-related protein, a marker of cytoplasmic droplets. We also demonstrate that curcumin significantly suppressed the increased expression of transforming growth factor $\beta$, vascular endothelial growth factor and extracellular matrix proteins such as type IV collagen and fibronectin. In addition, curcumin treatment increased nephrin expression to near-normal levels in diabetic rats. These results demonstrated that curcumin protects against the development of diabetic nephropathy through the AMPK-SREBP pathway and the reduction of renal triglyceride accumulation which could be a possible mechanism by which curcumin preserves renal function in diabetes.
\end{abstract}

(c) 2012 Elsevier Inc. All rights reserved.

Keywords: Curcumin; Diabetic nephropathy; AMPK; SREBP; Dyslipidemia; Streptozotocin-induced diabetes

\section{Introduction}

Diabetic nephropathy (DN) is characterized by early vascular dysfunction and increasing matrix accumulation in the kidney, eventually leading to proteinuria, glomerulosclerosis and interstitial fibrosis [1]. Since the description by Kimmelstiel and Wilson [2] of classical nodular glomerulosclerosis and the presence of lipid deposits in diabetic kidney, several investigators have shown the presence of lipid deposits in the kidney of diabetic humans and experimental animals, and they have proposed that these deposits may play an important role in the pathogenesis of diabetic kidney disease in addition to the notorious role played by hyperglycemia, hypertension, growth factors, inflammatory cytokines, oxidative stress and advanced glycation end products $[3,4]$. That the deposition of lipids per

This research was supported by a Yujin Memorial Grant, Ministry of Education, Culture, Sports and Technology of Japan, and by a grant from the Promotion and Mutual Aid Corporation for Private Schools, Japan.

* Corresponding author.

E-mail address: watanabe@nupals.ac.jp (K. Watanabe). se may mediate diabetic renal disease is supported by up-regulated renal expression of the transcriptional factor sterol regulatory element-binding protein (SREBP)-1c, which causes increased fatty acid synthesis and accumulation of triglycerides, lipid bodies and cholesterol in the kidneys $[5,6]$.

The SREBPs have been described as master regulators of both fatty acid and cholesterol metabolism [7-9]. Studies have shown that increased renal expression of a transcriptional factor, SREBP-1, resulted in increased synthesis and accumulation of triglyceride and was correlated with renal sclerosis and proteinuria $[6,10]$. The role of SREBP-1 in DN was demonstrated in a study using SREBP-1c knockout mice. The absence of SREBP-1c completely abolished the increase in urinary albumin excretion induced by diabetes in the streptozotocin (STZ)-treated mice, revealing the crucial role of SREBP-1c in DN [11].

Another important modulator of lipid metabolism is $5^{\prime}$ adenosine monophosphate (AMP)-activated protein kinase (AMPK). AMPK is a heterotrimeric enzyme complex consisting of a catalytic subunit $(\alpha)$ and two regulatory subunits ( $\beta$ and $\gamma$ ) [12]. Previous studies demonstrated that increased phosphorylation and activation of AMPK lead to suppression of SREBP-1c protein expression and, as a 
result, down-regulation of target genes of SREBP-1c which preferentially controls the expression of genes involved in triglyceride synthesis and accumulation, such as acetyl-CoA carboxylase (ACC) and fatty acid synthase (FAS) $[13,14]$. It has been reported that AMPK is regulated by cytokines in the control of whole-body energy balance, by antidiabetic drugs and by natural plant products such as berberine, resveratrol and green tea catechin epigallocatechin-3-gallate [12].

Previous investigations have shown that curcumin, a yellow coloring component of the rhizome of Curcuma longa L, has complex pharmacological effects, such as antiinflammatory, antioxidant, anticarcinogenic and hypolipidemic activities $[15,16]$. Study showed that some genes, such as low-density-lipoprotein receptor, SREBP and $\mathrm{CD} 36$, involved in cholesterol homeostasis were influenced by curcumin [17-20]. In an in vitro study, curcumin activated AMPK and suppressed hepatic gluconeogenic gene expression [21]. Furthermore, curcumin has been shown to protect against nephropathy, retinopathy and islet damage in STZ-induced diabetic rats [22,23]. However, the molecular mechanisms that mediate the triglyceridelowering activity of curcumin are poorly understood. In this study, we investigated the effect of curcumin on activating AMPK, decreasing tissue triglyceride as well as SREBP-1c expression in the kidney and on the attenuation of renal dysfunction in STZ-induced type 1 diabetes.

\section{Methods and materials}

\subsection{Animal model}

Male Sprague-Dawley rats (250-280 g; obtained from Charles River Japan Inc.) were housed in colony cages, maintained on a 12-h light/12-h dark cycle. Rats in the STZ group were injected intraperitoneally with $55 \mathrm{mg} / \mathrm{kg}$ body weight of $20 \mathrm{mM}$ sodium citrate solution ( $\mathrm{pH} 4.5$ ), and rats in the control group were injected with $20 \mathrm{mM}$ sodium citrate solution. Tail vein blood glucose levels were measured every week, and rats with blood glucose of greater than $300 \mathrm{mg} / \mathrm{dl}$ were considered diabetic. After 3 weeks of induction of diabetes, rats were randomized to receive either vehicle or curcumin ( $100 \mathrm{mg} / \mathrm{kg}$ body weight; Sigma-Aldrich, Tokyo, Japan) by oral gavage and followed for 8 weeks ( $n=5$ per group). Curcumin was suspended in $0.5 \%$ carboxymethyl cellulose. Before sacrifice, individual rats were placed in metabolic cages to collect 24-h urine samples for the measurement of urine protein and creatinine concentrations, which were determined by the Bradford and Jaffe methods, respectively $[24,25]$. All animal studies were performed in accordance with protocols approved by the Institutional Animal Care and Use Committee of Niigata University.

\subsection{Estimation of biochemical parameters}

Blood samples were drawn via heart puncture in each rat at the time of death into EDTA Vacutainer tubes. EDTA blood was centrifuged at $3000 \mathrm{~g}, 4^{\circ} \mathrm{C}$, for $15 \mathrm{~min}$ for separation of plasma. The plasma was used for the estimation of plasma lipids and creatinine. Creatinine clearance was calculated in individual rats as follows: creatinine clearance $=$ urine creatinine $\times$ urine volume/plasma creatinine $\times$ time [26].

\subsection{Lipid extraction and triglyceride analysis}

Briefly, total lipids were extracted from rat kidney by the method of Bligh and Dyer [27]. Triglyceride in the kidneys was analyzed using a triglyceride determination kit.

\subsection{Preparation of kidney homogenates, membrane fraction and nuclear extracts}

Kidney cortex was homogenized in ice-cold buffer A ( 20 mmol/L Tris- $\mathrm{HCl}, \mathrm{pH} 7.5 ; 2$ $\mathrm{mmol} / \mathrm{L}$ EDTA; $10 \mathrm{mmol} / \mathrm{L}$ EGTA; and $0.25 \mathrm{~mol} / \mathrm{L}$ sucrose containing complete protease and phosphatase inhibitors). To prepare membrane fractions, tissue homogenates were centrifuged at $1000 \mathrm{~g}$ for $10 \mathrm{~min}$ at $4^{\circ} \mathrm{C}$, and the supernatant was then ultracentrifuged at $100,000 \mathrm{~g}$ for $1 \mathrm{~h}$ at $4^{\circ} \mathrm{C}$. This supernatant was retained as the cytosolic fraction, and the pellet was resuspended in buffer B (buffer A with $1 \%$ Triton $\mathrm{X}-100$ ) and ultracentrifuged at $100,000 \mathrm{~g}$ for $1 \mathrm{~h}$ at $4^{\circ} \mathrm{C}$. This supernatant was retained as the membranous fraction [28]. To prepare nuclear fraction, kidney cortex was homogenized in ice-cold HEPES buffer (10 mM HEPES, $0.2 \%$ Triton X-100, $50 \mathrm{mM} \mathrm{NaCl}$, $0.5 \mathrm{mM}$ sucrose, $0.1 \mathrm{mM}$ EDTA, and protease and phosphatase inhibitors) and homogenized with an ice-chilled Dounce homogenizer at $4^{\circ} \mathrm{C}$. This was spun at 10,000 rpm for $10 \mathrm{~min}$. The pellet was suspended in ice-cold buffer ( $10 \mathrm{mM}$ HEPES, $500 \mathrm{mM}$ $\mathrm{NaCl}, 10 \%$ glycerol, $0.1 \mathrm{mM}$ EDTA, $0.1 \mathrm{mM}$ EGTA, 0.1\% IGEPAL, and protease and phosphatase inhibitors), vortexed at $4^{\circ} \mathrm{C}$ for $15 \mathrm{~min}$ and centrifuged for $10 \mathrm{~min}$ at $14,000 \mathrm{rpm}$. The resulting supernatant was aliquoted and stored as the nuclear extract at $-80^{\circ} \mathrm{C}$. The absence of cross-reactivity with $\beta$-actin in Western blots confirmed the purity of nuclear extracts. A small aliquot of kidney homogenate, membrane fraction and nuclear extract was kept at $4^{\circ} \mathrm{C}$ for protein estimation [29].

\subsection{Western blot analyses}

Protein samples were subjected to sodium dodecyl sulfate polyacrylamide gel electrophoresis and then transferred to nitrocellulose membranes. Membranes were blocked in $5 \%$ powdered milk in Tris-buffered saline with Tween $(0.2 \%$ Tween 20 in $1 \times$ Tris-buffered saline) (TBS-T) and incubated using the following antibodies: Antibodies against SREBP-1c, AMPK $\alpha$, p-AMPK $\alpha$ (Thr 172), adipose differentiation-related protein (ADRP) and vascular endothelial growth factor (VEGF) were purchased from Santa Cruz Biotechnology, Inc. (Santa Cruz, CA, USA). Antibody against transforming growth factor (TGF)- $\beta$ was obtained from Promega. Antibody against nephrin was obtained from ProSci. All antibodies were used at a dilution of 1:1000. After washing for three times with TBS-T, incubation with appropriate horseradish-peroxidase-conjugated secondary antibodies was performed for $1 \mathrm{~h}$ at room temperature. Next, samples were washed three times with $1 \times$ Tris-buffered saline and then developed using a chemiluminescence detection system (Amersham Biosciences, Buckinghamshire, UK). The signals were quantified with densitometric analysis using Scion Image program (Epson GT-X700, Tokyo, Japan). Antibodies to Lamin A (Santa Cruz) and $\beta$ actin (Cell Signaling) served as housekeeping proteins for nuclear and cytosolic target proteins, respectively.

\subsection{Quantitative real-time polymerase chain reaction $(P C R)$}

Kidney tissues were preserved by immersion in RNAlater (Ambion Inc., Austin, TX, USA) immediately after sampling. Total RNA was extracted after homogenization using Ultra TurraxT8 (IKA Labortechinik, Staufen, Germany) in TRIzol reagent (Invitrogen Corp., Carlsbad, CA, USA) according to the standard protocol. Then, cDNA was synthesized with Invitrogen SuperScript preamplification system. To investigate the expression of ACC and FAS mRNA, real-time PCR (QIAcube real-time analyzer, Qiagen, Germantown, MD, USA) was performed with TaqMan probe (TaqMan Gene expression assays; Applied Biosystems, Foster City, CA, USA) according to the following protocol: $95^{\circ} \mathrm{C}$ for $3 \mathrm{~min}, 95^{\circ} \mathrm{C}$ for $15 \mathrm{~s}, 60^{\circ} \mathrm{C}$ for $30 \mathrm{~s}, 72^{\circ} \mathrm{C}$ for $30 \mathrm{~s}, 40$ cycles. Primer sequences were as follows: ACC (forward), CCCAGCAGAATAAAGCTACTTTGG, (reverse), TCCTTTTGTGCAACTAGGAACGT; FAS, (forward), CCTGGATAGCATTCCGAACCT, (reverse), AGCACATCTCGAAGGCTACACA; GAPDH, (forward), GCTCATTTCCTGGTATGACAACG, (reverse), AGGGGTCTACATGGCAACTG. Relative standard curves representing several 10-fold dilutions $(1: 10: 100: 1000: 10,000: 100,000)$ of cDNA from kidney tissue samples were used for linear regression analysis of other samples. Results were normalized to GAPDH mRNA as an internal control and are thus shown as relative mRNA levels.

\subsection{Histology and oil red 0 staining}

Paraffin sections were stained with periodic acid-Schiff, Azan-Mallory, and hematoxylin and eosin (H\&E). The stained kidney sections were imaged with an Olympus microscope, and 100 glomeruli showing global and/or segmental sclerosis and tubules showing dilatation and epithelial hyperplasia were scored semiquantitatively in a blinded manner. Frozen sections were used for oil red $\mathrm{O}$ staining to determine renal accumulation of neutral fats [30]. Briefly, kidney transverse frozen sections were cut at $8 \mu \mathrm{m}$ and placed on glass slides. To determine lipid deposition, sections were fixed in $10 \%$ formalin for $10 \mathrm{~min}$, rinsed in distilled water and stained with oil red O solution (Muto Pure Chemicals, Tokyo, Japan) which was preheated to $60^{\circ} \mathrm{C}$, for $5 \mathrm{~min}$. Sections were rinsed with $60 \%$ isopropanol for $2 \mathrm{~min}$ and distilled water and counterstained with Mayer's hematoxylin for $1 \mathrm{~min}$. These were subsequently used to assess the area of lipid deposition.

\subsection{Immunofluorescence staining for fibronectin, type IV collagen and ADRP}

Immunofluorescence for type IV collagen and fibronectin was done on frozen sections. Briefly, frozen sections were preincubated for $10 \mathrm{~min}$ at room temperature with $3 \%$ milk powder in phosphate-buffered saline containing $0.05 \%$ Triton X-100. Nonspecific binding sites were blocked with $2 \%$ goat serum for 60 min and washed for three times for 5 min each. They were then incubated with rabbit polyclonal serum against type IV collagen (1:50; Abcam, Cambridge, England) and mouse monoclonal serum against fibronectin $\left(1: 50\right.$; Santa Cruz, CA, USA) at $1: 500$ overnight at $4^{\circ} \mathrm{C}$. The sections were rinsed three times with phosphate-buffered saline before incubation for $1 \mathrm{~h}$ with the secondary antibody, fluorescein isothiocyanate-conjugated (FITC) goat anti-rabbit $\operatorname{IgG}$, at $1: 100$ in the dark at room temperature. After rinsing with phosphate-buffered saline, the sections were mounted using 3:1 Vectashield:DAPI. The kidney sections were then imaged with a fluorescence microscope at 400 -fold magnification (CIA-102; Olympus) [5]. For ADRP imaging, paraffin-embedded sections were used. The sections were deparaffinized in xylene and rehydrated in graded ethanol and distilled water, and the antigen was unmasked with sodium citrate (10 $\mathrm{mM}, \mathrm{pH} 6.0$ ), followed by microwave heat source on high power for $3 \mathrm{~min}$ and then with low power for $10 \mathrm{~min}$. After phosphate-buffered saline wash, the nonspecific background was blocked with 3\% normal rabbit serum at room temperature for $1 \mathrm{~h}$. Incubation with anti-ADRP (1:50; Santa Cruz, CA, USA) was performed at room 
temperature for $60 \mathrm{~min}$. Sections were then washed in phosphate-buffered saline and incubated with FITC-conjugated rabbit anti-goat secondary antibody (1:50; SigmaAldrich, Tokyo, Japan). After extensive washing with phosphate-buffered saline, slides were mounted in 3:1 Vectashield:DAPI and examined with an Olympus CIA-102 fluorescence microscope equipped with an Olympus camera. The integrated fluorescence intensity was quantified and measured with the HPIAS-1000 ImageAnalysis System. The relative fluorescence intensity was calculated by dividing the total integrated optical density by the total number of cells in each field. Mean fluorescence intensity measurements were obtained from six separate experiments in each group [31-33].

\subsection{Statistical analysis}

Data are shown as mean + S.E.M. and were analyzed using one-way analysis of variance followed by Tukey's methods for post hoc analysis and two-tailed $t$ test when appropriate. A value of $P<.05$ was considered statistically significant. For statistical analysis, GraphPad Prism 5 software (San Diego, CA, USA) was used.

\section{Results}

\subsection{Biochemical parameters in experimental animals}

As shown in Table 1, the mean plasma glucose levels were significantly higher in vehicle-treated diabetic rats than in control nondiabetic rats $(P<.01)$. Chronic treatment with curcumin in diabetic rats altered plasma glucose levels significantly compared with those in vehicle-treated diabetic rats. To evaluate the effect of curcumin on preventing hyperfiltration induced by STZ, we measured 24-h urinary protein excretion. Although vehicle-treated diabetic rats showed a marked elevation of 24-h urinary protein excretion, curcumin treatment significantly improved this alteration. Moreover, plasma triglyceride was significantly increased in the vehicle-treated diabetic rats compared with that in the control nondiabetic rats. The administration of curcumin markedly decreased plasma triglyceride levels. In addition, vehicle-treated diabetic rats also exhibited increased plasma creatinine and plasma urea, and decreased creatinine clearance, which were all ameliorated by curcumin treatment. At 11 weeks of STZ-induced type 1 diabetes, kidney weight corrected for body weight was significantly increased by $45 \%$ in vehicle-treated diabetic rats (Fig. $1 \mathrm{~A} ; P<.01$ ), which was prevented by curcumin treatment.

\subsection{Curcumin inhibits lipid accumulation in kidney}

There were significant increases in renal triglyceride content in vehicle-treated diabetic rats, which were reversed by curcumin treatment (Fig. 1B). ADRP is a marker of lipid droplets. Western blot analysis clearly showed a 2.2-fold increase of ADRP protein expression in vehicle-treated diabetic rats (Fig. 2A), which was attenuated by curcumin treatment. In agreement with Western blot analysis, immunofluorescence staining of ADRP revealed typical lipid droplets as ring-shaped in the tubules of vehicle-treated diabetic rats (Fig. 2C). In contrast, there were almost no lipid droplets in the

Table 1

Biochemical parameters of the experimental animals at the end of intervention study $(n=5)$

\begin{tabular}{|c|c|c|c|}
\hline & $\mathrm{C}$ & $\mathrm{D}$ & Cur \\
\hline 24-h urine protein (g/day) & $12.5 \pm 0.7$ & $42 \pm 5.2^{*}$ & $15.2 \pm 3.7^{* * *}$ \\
\hline Plasma glucose $(\mathrm{mg} / \mathrm{dl})$ & $118.6 \pm 6$ & $744.4 \pm 9.3^{*}$ & $597.1 \pm 9.9^{*}, * * *$ \\
\hline Plasma urea $(\mathrm{mg} / \mathrm{dl})$ & $21.9 \pm 0.6$ & $45 \pm 1.9^{*}$ & $33.2 \pm 2.5^{*}, * * *$ \\
\hline Plasma creatinine $(\mathrm{mg} / \mathrm{dl})$ & $0.3 \pm 0.01$ & $2.1 \pm 0.1^{*}$ & $0.8 \pm 0.2^{* *}, * * *$ \\
\hline Creatinine clearance $(\mathrm{ml} / \mathrm{min})$ & $3.9 \pm 0.5$ & $0.8 \pm 0.1^{*}$ & $2.7 \pm 0.5^{*}, * * *$ \\
\hline Plasma triglyceride (mg/dl) & $104.8 \pm 2.1$ & $320.3 \pm 2.1^{*}$ & $114 \pm 3.7^{* * *}$ \\
\hline
\end{tabular}

\footnotetext{
Data are means \pm S.E.M

* $P<.01$.

** $P<.05$ vs. C.

*** $P<.01$ vs. D.
}
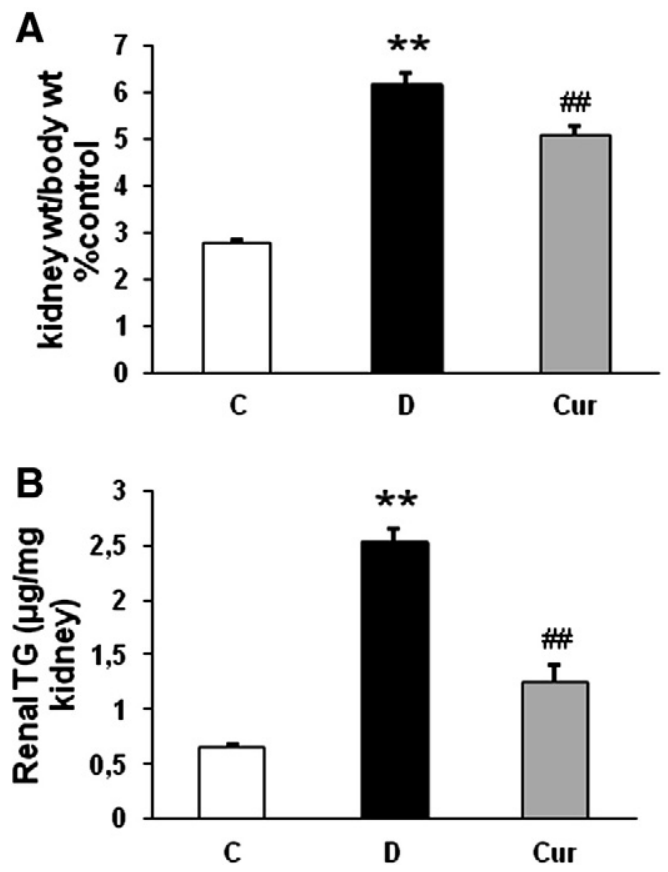

Fig. 1. (A) Effect of curcumin treatment on the kidney weight to body weight ratio. (B) The vehicle-treated diabetic rats showed statistically significant increases in renal triglyceride content, which were reduced by curcumin treatment. Results are expressed as mean \pm S.E.M. $n=5$ per group. C, age-matched normal rats; D, diabetictreated rats administered with vehicle; Cur, diabetic rats treated with curcumin $100 \mathrm{mg} / \mathrm{kg} /$ day. ${ }^{* *} P<.01$ vs. C; ${ }^{\# \#} P<.01$ vs. D.

curcumin-treated diabetic rats (Fig. 2D). Likewise, there is very strong staining of oil red $\mathrm{O}$ in the glomerular and tubulointerstitial cells of vehicle-treated diabetic rats, which indicates accumulation of neutral lipids deposits (Fig. 2F) and almost no staining in the curcumintreated diabetic rats (Fig. 2G). Lipid accumulation was also shown by the unstained area in renal tissue under H\&E staining in the vehicletreated diabetic rats (Fig. 2I) which was reduced by curcumin treatment (Fig. 2J). These data strongly indicate that there were excessive amounts of lipid deposits in the kidneys of vehicle-treated diabetic rats. Curcumin treatments improved this lipid accumulation in the kidneys of diabetic rats.

\subsection{Renal histologic change in experimental animals}

Periodic acid-Schiff staining reveals $\sim 56 \%$ sclerosis of glomeruli in vehicle-treated diabetic rats (Fig. $2 \mathrm{~L}$ ), whereas the curcumin-treated diabetic rats developed only $28.3 \%(P<.05)$ (Fig. $2 \mathrm{M}$ ) when compared with control nondiabetic rats (Fig. $2 \mathrm{~K}$ ). In addition to pronounced glomerular sclerosis, severe tubulointerstitial changes were observed in the vehicle-treated diabetic rats. Histological evaluations revealed that the vehicle-treated diabetic rats had 58.3\% dilated tubules (Fig. 2L). Curcumin treatment significantly reduced this to $34 \%$ $(P<.05)$ compared with that in vehicle-treated diabetic rats (Fig. $2 \mathrm{M})$. Interstitial collagen deposition was studied in Azan-Mallory-stained renal sections as an index of interstitial fibrosis. Vehicle-treated diabetic rats showed higher interstitial fibrosis (Fig. 20) than control nondiabetic rats (Fig. $2 \mathrm{~N}$ ), which was improved in the curcumintreated diabetic rats (Fig. 2P). Immunofluorescence microscopy with anti-fibronectin and anti-type IV collagen antibodies revealed increased intensity of immunofluorescence in the glomeruli and tubulointerstitial cells in vehicle-treated diabetic rats (Fig. 2R, 2U), indicating accumulation of extracellular matrix (ECM) proteins and glomerulosclerosis and tubulointerstitial fibrosis, whereas curcumin 

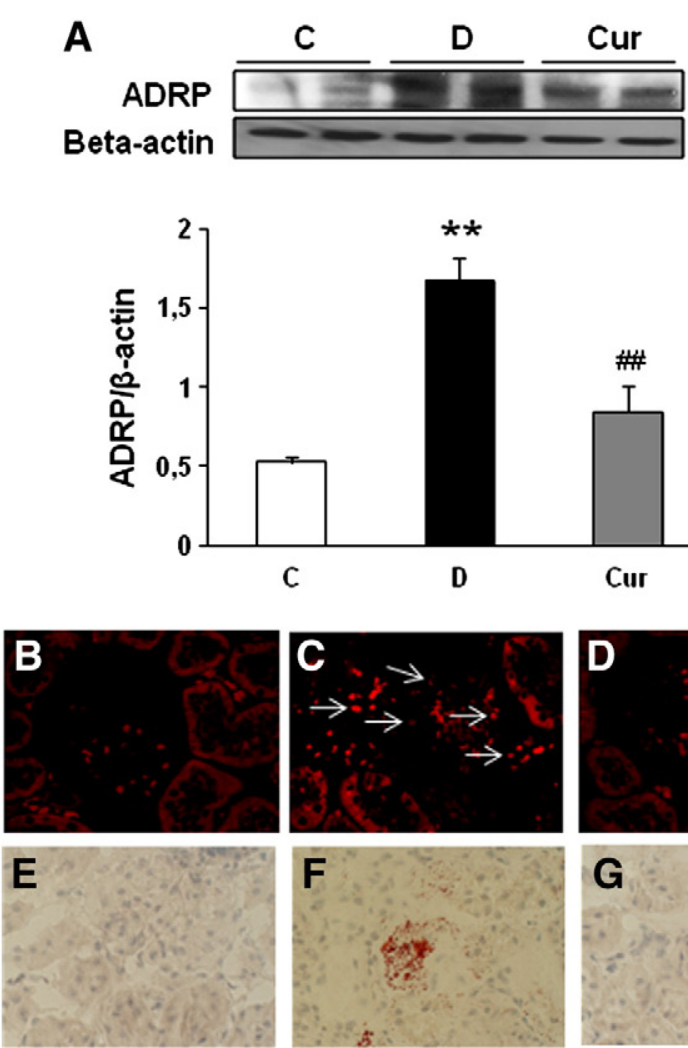
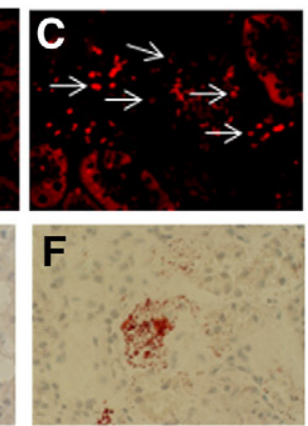
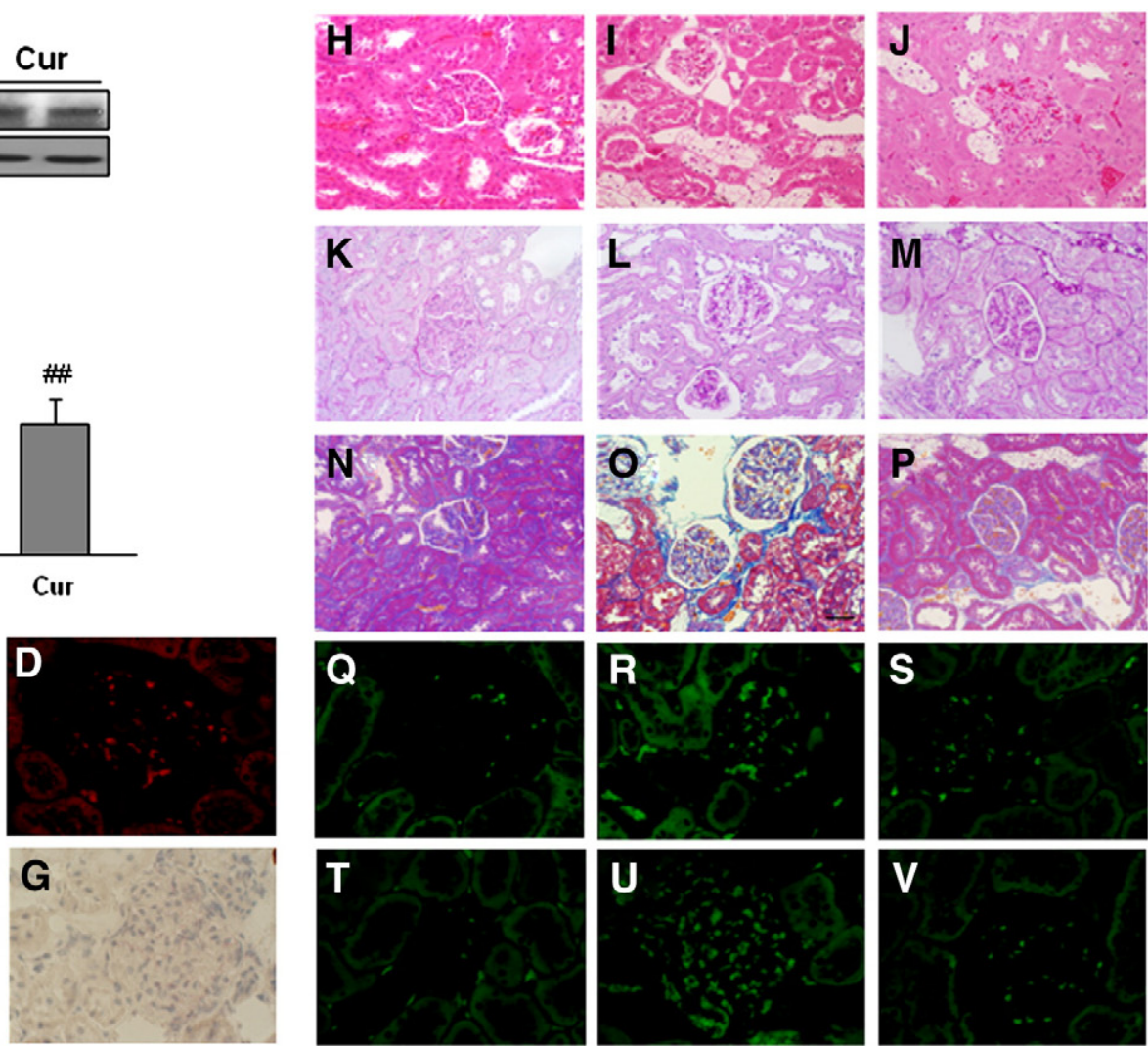
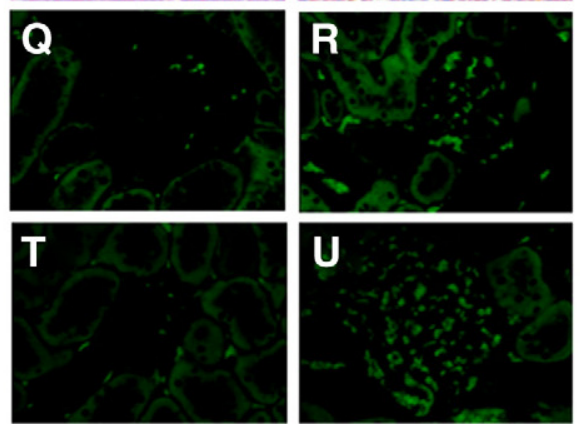
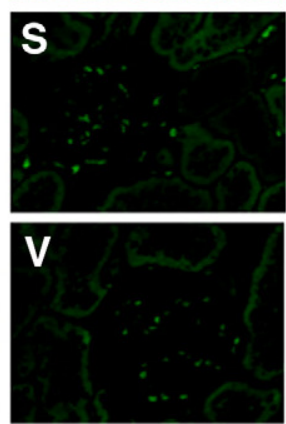

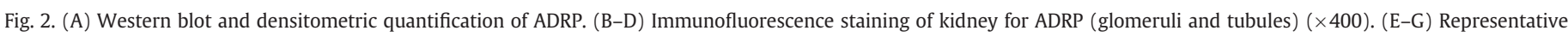

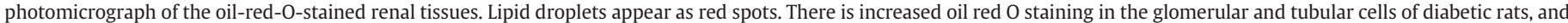

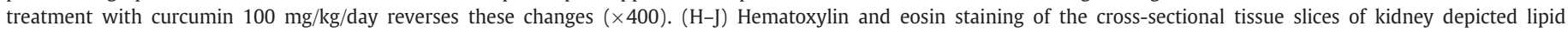

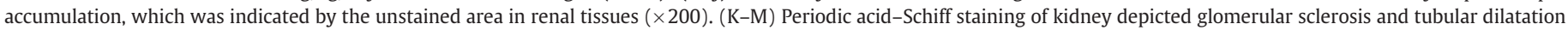

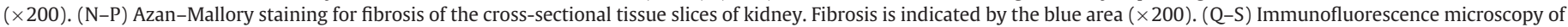

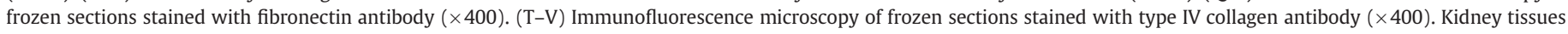

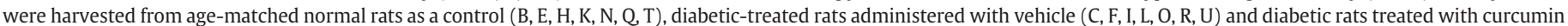
$100 \mathrm{mg} / \mathrm{kg} /$ day $(\mathrm{D}, \mathrm{G}, \mathrm{J}, \mathrm{M}, \mathrm{P}, \mathrm{S}, \mathrm{V}) . n=5$ rats in each experimental group. ${ }^{* *} P<.01$ vs. C; ${ }^{\# \#} P<.05$ vs. D.

suppressed the increased intensity of fibronectin and type IV collagen expression in the diabetic rats (Fig. 2S, V).

\subsection{Effect of curcumin on renal expression of AMPK}

To understand the mechanism by which curcumin attenuated lipid accumulation in the kidneys of diabetic rats, we measured the effect of curcumin on AMPK activation on renal tissues. Since phosphorylation of the catalytic subunit AMPK- $\alpha$ at Thr172 position is essential for AMPK activation, AMPK activation was monitored by using a specific antibody that recognizes the phosphorylated AMPK- $\alpha$ at Thr172. As shown in Fig. 3A, AMPK phosphorylation was reduced in the vehicle-treated diabetic rats by nearly $60 \%(P<.01)$. Curcumin prevented the reduction in AMPK phosphorylation in diabetic rats, suggesting that it may protect the AMPK activity.

3.5. Effect of curcumin on renal expression of nuclear receptors and genes that regulate triglyceride and cholesterol metabolism

SREBP-1c is a transcription factor that activates the transcription of genes involved in fatty acid and triglyceride synthesis, such as ACC and FAS [34]. To assess the SREBP-1c precursor and the nuclear form, cell lysates were fractionated into a separate membrane fraction containing SREBP precursors and a nuclear fraction containing active nSREBP [35]. Fig. 3 (B and C) shows that both precursor (2.6-fold) and mature forms (2.7-fold) of SREBP-1c were increased in the vehicle- treated diabetic rats. It is noteworthy that curcumin administration significantly reduced both precursor and mature forms of SREBP-1C and was well correlated with mRNA encoding of the SREBP-1c target genes, ACC and FAS (Fig. 4A and B).

\subsection{Curcumin decreases proteinuria and renal expression of profibrotic} growth factors

Because mesangial expansion and accumulation of ECM proteins are mediated by increased expression of growth factors such as TGF- $\beta$ [36] and VEGF [37], we measured the expression of TGF- $\beta 1$ and VEGF in the kidney cortex of experimental animals. There were a 1.8-fold increase in TGF- $\beta 1$ (Fig. 5A) and a 2.4-fold increase in VEGF (Fig. 5B) expression, and curcumin treatment ameliorated these increases by 1.2- and 1.4-fold, respectively. Next, we analyzed the expression pattern of the slit diaphragm protein nephrin. Fig. $5 \mathrm{C}$ shows that the down-regulation of nephrin occurred in the vehicle-treated diabetic rats compared with that in the control nondiabetic rats. This decrease in renal nephrin protein expression was markedly increased by curcumin treatment $(P<.05)$.

\section{Discussion}

The most important findings of this study were as follows: (1) Curcumin can activate AMPK by increasing AMPK phosphorylation in renal tissue in STZ-induced type 1 diabetes. (2) This increase of AMPK 

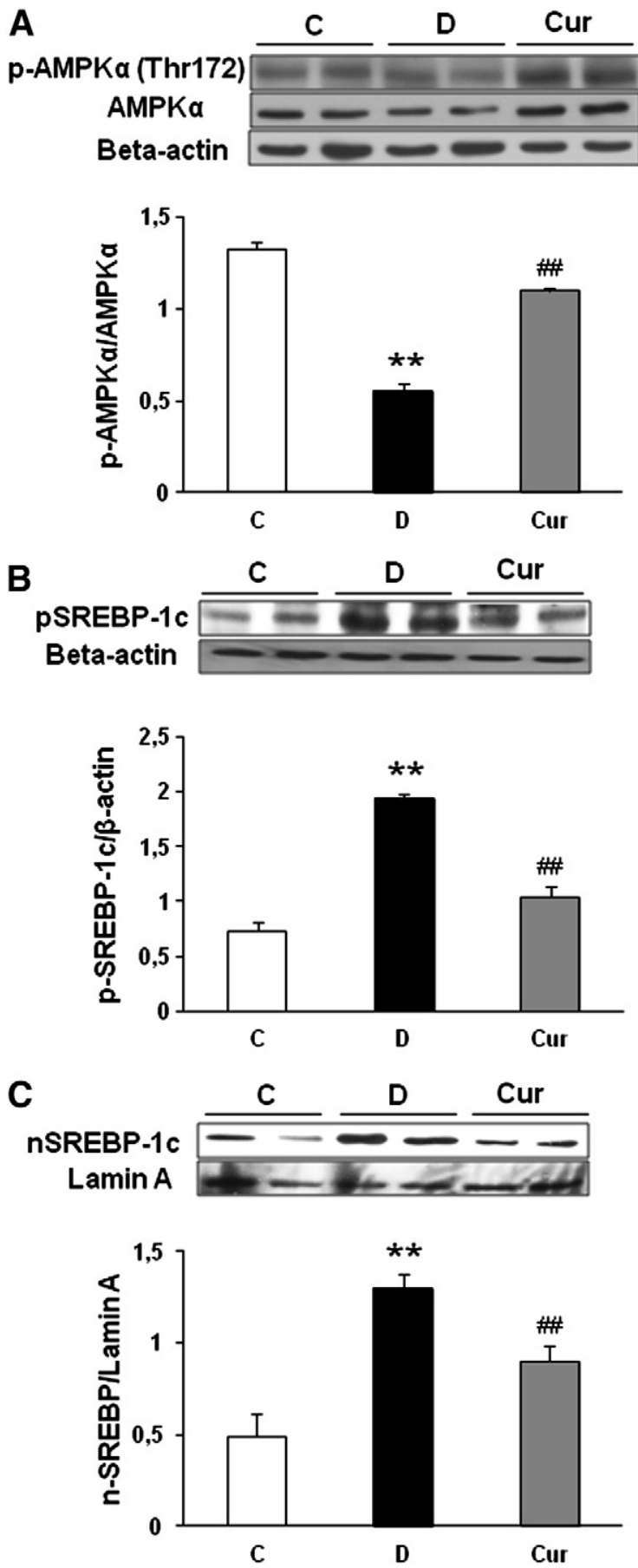

Fig. 3. (A) Representative Western blots and group data depicting protein abundance of the phosphorylated AMPK in the renal tissues. (B) Western blots analysis of the expression of SREBP-1c nuclear extracts and membrane (precursor) (C) isolated from kidney tissues. STZ-induced type 1 diabetes causes increased SREBP-1c expression in both nuclear and membrane extracts, and these changes were reversed upon treatment with curcumin. Data are means \pm S.E.M. $n=5$ per group. C, age-matched normal rats; D, diabetic-treated rats administered with vehicle; Cur, diabetic rats

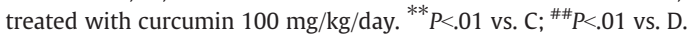

activation, consecutively, sharply suppresses renal SREBP-1c and the target genes such as ACC and FAS. (3) As a result, curcumin attenuates proteinuria, glomerulosclerosis, and tubulointerstitial dilatation and fibrosis. In the present study, we also found that, in STZ-induced type $1 \mathrm{DN}$ rats, there were excessive amounts of lipid deposits in the kidney sections, as shown by oil red $\mathrm{O}$ and H\&E staining, the presence of lipid bodies, as shown by ADRP immunofluorescence microscopy and ADRP protein levels, using Western blot analysis; and significantly higher renal content of triglyceride compared with those in control nondiabetic rats; all of these abnormalities were ameliorated by curcumin treatment.

Accumulating evidence indicates that curcumin (diferuloylmethane), a polyphenol natural product of the plant $C$. longa, exerts effects on a wide range of molecular targets controlling lipid accumulation and has antidiabetic and anticancer activities [15,16]. Our previous study in STZ-induced type 1 DN showed that curcumin exhibited renoprotective effects to lessen proteinuria and diabetic pathologic changes through inhibition of macrophage infiltration, which suggests that curcumin has an antifibrotic and anti-inflammatory effect [38]. Diabetic rats with curcumin diet showed that curcumin has a hypocholesterolemic action via stimulation of hepatic cholesterol-7a-hydroxylase and HMG-CoA reductase [16]. Curcumin also inhibits ox-LDL-induced cholesterol accumulation in cultured vascular smooth muscle cells through inhibition of nuclear translocation of SREBP-1 [18]. It has also been shown that curcumin improved muscular insulin resistance through the up-regulation of phosphorylated AMPK [39]. Furthermore, curcumin increases the phosphorylation of AMPK and its downstream target as well as suppresses gluconeogenic gene expression in hepatoma cells $[21,40]$. Although several lines of evidences strongly suggest that curcumin could regulate glucose and lipid homeostasis, the potential mechanism of curcumin in regulating triglyceride accumulation in type $1 \mathrm{DN}$ has not been studied. In the present study, we show for the first time that curcumin protects renal triglyceride accumulation in STZinduced type 1 diabetes through activation of AMPK and suppresses lipogenic gene expression.

AMPK is a heterotrimeric protein consisting of a combination of $\alpha_{1 / 2^{-}}, \beta_{1 / 2^{-}}$and $\gamma_{1 / 2 / 3^{-}}$subunits [41], with the $\alpha_{1^{-}}$rather than $\alpha_{2^{-}}$ subunit expressed in the kidney [42]. The enzymatic activity of AMPK is dependent on phosphorylation of Thr172 of the $\alpha$-subunit [43]. On
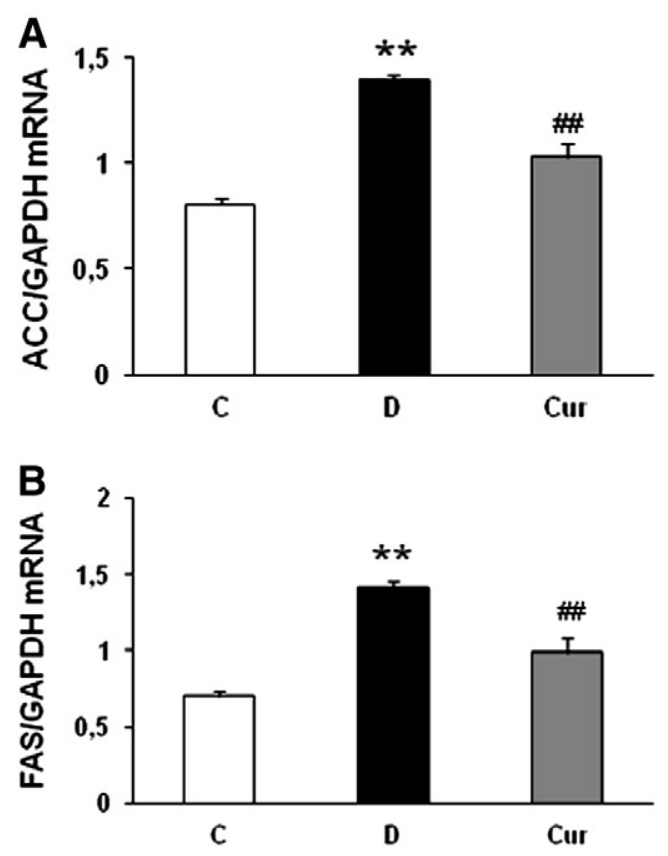

Fig. 4. (A and B) Effects of curcumin on renal mRNA levels of ACC and FAS in rats with DN were determined by quantitative reverse transcriptase PCR. The expression level of each sample is expressed relative to the expression level of GAPDH gene. Data are means \pm S.E.M. $n=5$ per group. C, age-matched normal rats; $D$, diabetic-treated rats administered with vehicle; Cur, diabetic rats treated with curcumin $100 \mathrm{mg} / \mathrm{kg} /$ day. ${ }^{* *} P<.01$ vs. C; ${ }^{\# \#} P<.05$ vs. D. 
A

\section{TGF- $\beta$}

Beta-actin

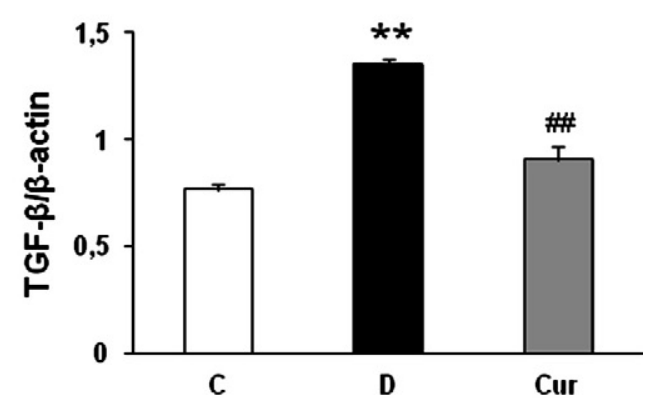

BEGF
Beta-actin

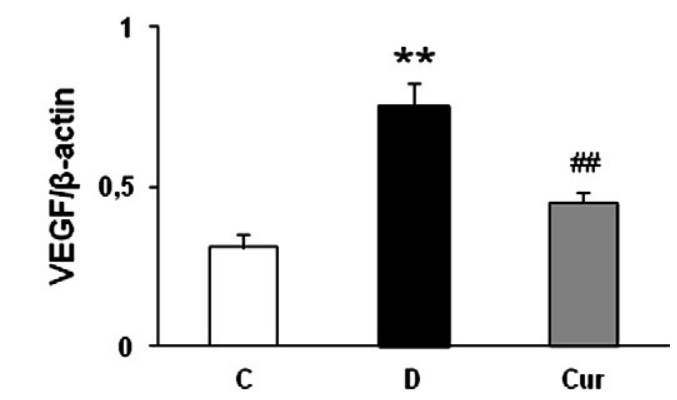

C
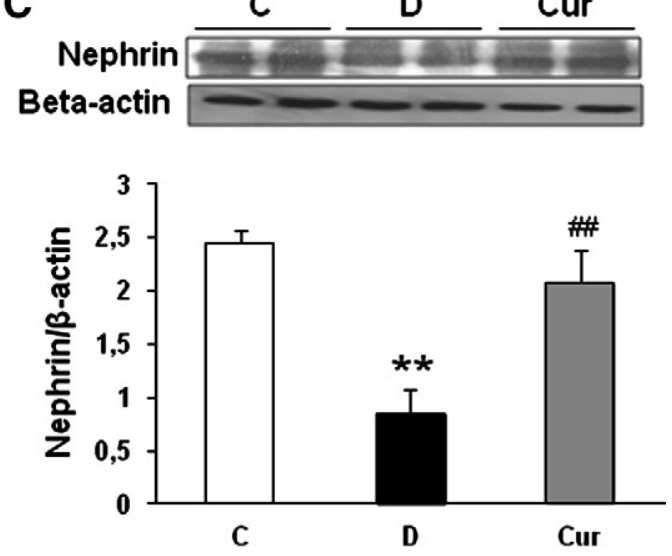

Fig. 5. Representative Western blots and group data depicted protein abundance of TGF- $\beta$ (A), VEGF (B) and nephrin (C) in the renal tissues of diabetic rats, curcumintreated diabetic rats and control nondiabetic rats. $n=5$ in each group. Data are

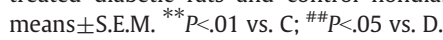

activation, AMPK inhibits energy-consuming reactions, such as synthesis of fatty acids and sterols, and activates adenosinetriphosphate-generating processes, such as fatty acid oxidation [44]. A very recent study also demonstrated that activation of AMPK inhibits hepatic fatty acid synthesis by suppressing SREBP-1c, a master regulator of lipogenic gene expression, in rat hepatoma cells [45]. Lee et al. have shown that, in STZ-induced insulin-deficient diabetes, a model of type 1 diabetes, AMPK phosphorylation was reduced at the time when the kidneys were hypertrophic [46]. In line with previous reports, we have also observed that both total and phosphorylated AMPK expressions were reduced in vehicle-treated diabetic rats (Fig. 3A) and that these decreases under diabetic conditions were ameliorated by curcumin treatment, which in turn attenuated the expression of SREBP-1c in the renal tissue of type 1 diabetic rats and, as a consequence, caused decreases in the mRNA abundance of two SREBP-1-activated enzymes that mediate fatty acid and triglyceride synthesis, ACC and FAS.

Abnormal lipid deposits have been observed in tubules of human diabetic kidney [47] and have been proposed to participate in the pathogenesis of DN and other proteinuric renal diseases [3]. Lipid droplets were also detected in the cortical tubules of the STZ-induced diabetic rats [5]. Increased deposition of lipid in the kidney contributes to cellular damage and to the progression of diabetic kidney disease. ADRP, also called adipophilin in humans, is a $\sim 50-\mathrm{kDa}$ fatty-acid-binding protein that is transcriptionally activated when preadipocytes differentiate into mature adipocytes [48]. ADRP belongs to the perilipin family of lipid storage proteins [49] and, similar to other perilipin members, localizes to the cytoplasmic surface of lipid storage droplets in distinct cell types. Induction of the ADRP gene is apparently an important aspect of the response to diabetes and formation of lipid storage droplets in the diabetic kidney. We found that the ADRP protein (Fig. 2A) and ADRP immunofluorescence (Fig. 2C) were significantly induced in vehicle-treated diabetic rat kidneys and corresponded with the presence of lipid droplets in oil red $\mathrm{O}$ (Fig. 2F) and H\&E staining (Fig. 2I) and increase in renal triglyceride content (Fig. 1B), and that these increases under diabetic conditions were ameliorated by curcumin treatment.

In vivo and in vitro studies have shown that low-density lipoprotein or very low density lipoprotein induces up-regulation of growth factors, including TGF- $\beta$ and platelet-derived growth factor, ECM [50], lipid peroxidation and glycoxidation [51], and change in expression of podocyte-specific proteins such as nephrin [52], indicating a direct role for lipids in activating the mediators of glomerulosclerosis and proteinuria. Ishigaki et al. indicated that activation of renal SREBP-1c was consistently associated with pathohistological fibrosis and TGF- $\beta$ activation both in vivo and in vitro, accompanying induction of fibrosis markers such as type IV collagen and fibronectin [11]. It has also been reported that renal hypertrophy in STZ-induced type 1 diabetic rats was associated with reduction in AMPK phosphorylation [53]. Our study indicates that accumulation of renal triglyceride in STZ-treated diabetic rats caused increased expression of TGF- $\beta$ and VEGF as well as ECM proteins such as type IV collagen and fibronectin and decreased expression of nephrin, resulting in glomerulosclerosis and proteinuria, respectively. Curcumin treatment markedly decreased TGF- $\beta$ and VEGF and increased nephrin expression in renal tissue of diabetic rats. Curcumin also reduced the augmented expressions of type IV collagen and fibronectin. This effect might be mediated by curcumin's function as an AMPK activator, which further down-regulated the SREBP-1c expression.

We have shown that curcumin inhibits renal triglyceride accumulation in STZ-induced type 1 DN by activation of AMPK and suppresses SREBP-1c expression, as well as inhibits the expression of lipogenic genes, which may explain some of the lipid-lowering effects of curcumin. Since AMPK is considered one of the preventive or therapeutic targets for metabolic diseases such as diabetes, obesity and cancer, the effective modulation of AMPK by curcumin could be a promising effective approach for treating these diseases.

\section{References}

[1] Catania JM, Chen G, Parrish AR. Role of matrix metalloproteinases in renal pathophysiologies. Am J Physiol Renal Physiol 2007;292:F905-11.

[2] Kimmelstiel P, Wilson C. Intercapillary lesions in the glomeruli of the kidney. Am J Pathol 1936;12:83-98.

[3] Keane WF. The role of lipids in renal disease: future challenges. Kidney Int Suppl 2000;75:S27-31. 
[4] Oda H, Keane WF. Lipids in progression of renal disease. Kidney Int Suppl 1997;62: S36-8.

[5] Sun L, Halaihel N, Zhang W, et al. Role of sterol regulatory element-binding protein 1 in regulation of renal lipid metabolism and glomerulosclerosis in diabetes mellitus. J Biol Chem 2002;277:18919-27.

[6] Wang Z, Jiang T, Li J, et al. Regulation of renal lipid metabolism, lipid accumulation, and glomerulosclerosis in FVBdb/db mice with type 2 diabetes. Diabetes 2005;54: 2328-35.

[7] Brown MS, Goldstein JL. The SREBP pathway: regulation of cholesterol metabolism by proteolysis of a membrane-bound transcription factor. Cell 1997; 89:331-40.

[8] Horton JD, Shimomura I. Sterol regulatory element-binding proteins: activators of cholesterol and fatty acid biosynthesis. Curr Opin Lipidol 1999;10:143-50.

[9] Rawson RB. Control of lipid metabolism by regulated intramembrane proteolysis of sterol regulatory element binding proteins (SREBPs). Biochem Soc Symp 2003;70:221-31.

[10] Proctor G, Jiang T, Iwahashi M, et al. Regulation of renal fatty acid and cholesterol metabolism, inflammation, and fibrosis in Akita and OVE26 mice with type 1 diabetes. Diabetes 2006;55:2502-9.

[11] Ishigaki N, Yamamoto T, Shimizu Y, et al. Involvement of glomerular SREBP-1c in diabetic nephropathy. Biochem Biophys Res Commun 2007;364:502-8.

[12] Hardie DG. AMP-activated protein kinase as a drug target. Annu Rev Pharmacol Toxicol 2007:47:185-210.

[13] Zhou G, Myers R, Li Y, et al. Role of AMP-activated protein kinase in mechanism of metformin action. J Clin Invest 2001;108:1167-74.

[14] Yang J, Craddock L, Hong S, et al. AMP-activated protein kinase suppresses LXRdependent sterol regulatory element-binding protein-1c transcription in rat hepatoma McA-RH7777 cells. J Cell Biochem 2009;106:414-26.

[15] Goel A, Kunnumakkara AB, Aggarwal BB. Curcumin as "Curecumin": from kitchen to clinic. Biochem Pharmacol 2008;75:787-809.

[16] Babu PS, Srinivasan K. Hypolipidemic action of curcumin, the active principle of turmeric (Curcuma longa) in streptozotocin induced diabetic rats. Mol Cell Biochem 1997;166:169-75.

[17] Peschel D, Koerting R, Nass N. Curcumin induces changes in expression of genes involved in cholesterol homeostasis. J Nutr Biochem 2007;18:113-9.

[18] Yuan HY, Kuang SY, Zheng X, et al. Curcumin inhibits cellular cholesterol accumulation by regulating SREBP-1/caveolin-1signaling pathway in vascular smooth muscle cells. Acta Pharmacol Sin 2008;29:555-63.

[19] Fan C, Wo X, Dou X, et al. Regulation of LDL receptor expression by the effect of curcumin on sterol regulatory element pathway. Pharmacol Rep 2006;58:577-81.

[20] Dou X, Fan C, Wo L, et al. Curcumin up-regulates LDL receptor expression via the sterol regulatory element pathway in HepG2 cells. Planta Med 2008;74:1374-9.

[21] Kim T, Davis J, Zhang AJ, et al. Curcumin activates AMPK and suppresses gluconeogenic gene expression in hepatoma cells. Biochem Biophys Res Commun 2009;388:377-82.

[22] Kowluru RA, Kanwar M. Effects of curcumin on retinal oxidative stress and inflammation in diabetes. Nutr Metab (Lond) 2007:4:8.

[23] Meghana K, Sanjeev G, Ramesh B. Curcumin prevents streptozotocin-induced islet damage by scavenging free radicals: a prophylactic and protective role. Eur J Pharmacol 2007;577:183-91.

[24] Bradford MM. A rapid and sensitive method for the quantitation of microgram quantities of protein utilizing the principle of protein-dye binding. Anal Biochem 1976;72:248-54.

[25] Husdan H, Rapoport A. Estimation of creatinine by the Jaffe reaction. A comparison of three methods. Clin Chem 1968;14:222-38.

[26] Bagheri F, Gol A, Dabiri S, et al. Preventive effect of garlic juice on renal reperfusion injury. Iran J Kidney Dis 2011;5:194-200.

[27] Bligh EG, Dyer WJ. A rapid method of total lipid extraction and purification. Can J Biochem Physiol 1959;37:911-7.

[28] Thallas-Bonke V, Thorpe SR, Coughlan MT, et al. Inhibition of NADPH oxidase prevents advanced glycation end product-mediated damage in diabetic nephropathy through a protein kinase C-alpha-dependent pathway. Diabetes 2008;57: 460-9.

[29] Ghosh SS, Massey HD, Krieg R, et al. Curcumin ameliorates renal failure in 5/6 nephrectomized rats: role of inflammation. Am J Physiol Renal Physiol 2009;296: F1146-57.
[30] Jiang T, Wang Z, Proctor G, et al. Diet-induced obesity in C57BL/6] mice causes increased renal lipid accumulation and glomerulosclerosis via a sterol regulatory element-binding protein-1c-dependent pathway. J Biol Chem 2005;280: 32317-25.

[31] Gao J, Serrero G. Adipose differentiation related protein (ADRP) expressed in transfected COS-7 cells selectively stimulates long chain fatty acid uptake. J Bio Chem 1999;274:16825-30.

[32] Xia Z, Kuo KH, Nagareddy PR, et al. N-acetylcysteine attenuates PKCbeta2 overexpression and myocardial hypertrophy in streptozotocin-induced diabetic rats. Cardiovasc Res 2007;73:770-82.

[33] McManaman JL, Palmer CA, Wright RM, et al. Functional regulation of xanthine oxidoreductase expression and localization in the mouse mammary gland: evidence of a role in lipid secretion. J Physiol 2002;545:567-79.

[34] Shimano H. Sterol regulatory element-binding proteins (SREBPs): transcriptional regulators of lipid synthetic genes. Prog Lipid Res 2001;40:439-52.

[35] DeBose-Boyd RA, Ou J, Goldstein JL, et al. Expression of sterol regulatory elementbinding protein 1c (SREBP-1c) mRNA in rat hepatoma cells requires endogenous LXR ligands. Proc Natl Acad Sci U S A 2001;98:1477-82.

[36] Basile DP. The transforming growth factor beta system in kidney disease and repair: recent progress and future directions. Curr Opin Nephrol Hypertens 1999;8:21-30.

[37] de Vriese AS, Tilton RG, Elger M, et al. Antibodies against vascular endothelial growth factor improve early renal dysfunction in experimental diabetes. J Am Soc Nephrol 2001;12:993-1000

[38] Soetikno V, Sari FR, Veeraveedu PT, et al. Curcumin ameliorates macrophage infiltration by inhibiting NF-kB activation and proinflammatory cytokines in streptozotocin induced-diabetic nephropathy. Nutr Metab (Lond) 2011;8:35

[39] Na LX, Zhang YL, Li Y, et al. Curcumin improves insulin resistance in skeletal muscle of rats. Nutr Metab Cardiovasc Dis 2011;21:526-33.

[40] Kanitkar M, Gokhale K, Galande S, et al. Novel role of curcumin in the prevention of cytokine-induced islet death in vitro and diabetogenesis in vivo. Br J Pharmacol 2008;155:702-13.

[41] Carling D. The AMP-activated protein kinase cascade-a unifying system for energy control. Trends Biochem Sci 2004;29:18-24.

[42] Fraser S, Mount P, Hill R, et al. Regulation of the energy sensor AMP-activated protein kinase in the kidney by dietary salt intake and osmolality. Am J Physiol Renal Physiol 2005;288:F578-86.

[43] Hawley SA, Davison M, Woods A, et al. Characterization of the AMP-activated protein kinase kinase from rat liver and identification of threonine 172 as the major site at which it phosphorylates AMP-activated protein kinase. J Biol Chem 1996;271:27879-87.

[44] Merrill GF, Kurth EJ, Hardie DG, et al. AICA riboside increases AMP-activated protein kinase, fatty acid oxidation, and glucose uptake in rat muscle. Am J Physiol 1997;273:E1107-12.

[45] Yap F, Craddock L, Yang J. Mechanism of AMPK suppression of LXR-dependent Srebp-1c transcription. Int J Biol Sci 2011;7:645-50.

[46] Lee MJ, Feliers D, Mariappan MM, et al. A role for AMP-activated protein kinase in diabetes-induced renal hypertrophy. Am J Physiol Renal Physiol 2007;292: F617-27.

[47] Ong AC, Moorhead JF. Tubular lipidosis: epiphenomenon or pathogenetic lesion in human renal disease? Kidney Int 1994;45:753-62.

[48] Jiang HP, Serrero G. Isolation and characterization of a full-length cDNA coding for an adipose differentiation-related protein. Proc Natl Acad Sci U S A 1992;89: 7856-60.

[49] Londos C, Brasaemle DL, Schultz CJ, et al. Perilipins, ADRP, and other proteins that associate with intracellular neutral lipid droplets in animal cells. Semin Cell Dev Biol 1999;10:51-8.

[50] Nishida Y, Oda H, Yorioka N. Effect of lipoproteins on mesangial cell proliferation. Kidney Int Suppl 1999;71:S51-3.

[51] Lee HS, Kim BC, Kim YS, et al. Involvement of oxidation in LDL-induced collagen gene regulation in mesangial cells. Kidney Int 1996;50:1582-90.

[52] Garman JH, Mulroney S, Manigrasso M, et al. Omega-3 fatty acid rich diet prevents diabetic renal disease. Am J Physiol Renal Physiol 2009;296:F306-16.

[53] Lee MJ, Feliers D, Sataranatarajan K, et al. Resveratrol ameliorates high glucoseinduced protein synthesis in glomerular epithelial cells. Cell Signal 2010;22: $65-70$. 